\title{
Antibiotic Sensitivity Profile of Microorganisms Isolated from Foods Sold in Selected Elementary Schools in Ilorin Metropolis
}

\author{
Alabede Iyabode Mubarakat , Henry Olawale Sawyerr, Habeeb Modupe Lateefat, \\ Adiama Babatunde Yusuf, Yusuf Olanrewaju Rauf \\ Department of Environmental Health Science, School of Allied Health and Environmental Science, Kwara State University, Malete, Nigeria
}

Email address:

alabede.mubarakat@gmail.com (A. I. Mubarakat)

${ }^{*}$ Corresponding author

\section{To cite this article:}

Alabede Iyabode Mubarakat, Henry Olawale Sawyerr, Habeeb Modupe Lateefat, Adiama Babatunde Yusuf, Yusuf Olanrewaju Rauf. Antibiotic Sensitivity Profile of Microorganisms Isolated from Foods Sold in Selected Elementary Schools in Ilorin Metropolis. Journal of Health and Environmental Research. Vol. 4, No. 2, 2018, pp. 51-55. doi: 10.11648/j.jher.20180402.12

Received: April 22, 2018; Accepted: May 15, 2018; Published: June 2, 2018

\begin{abstract}
Globally, the estimated amount of food found to be contaminated from schools particularly in developing countries keeps increasing. As a result of this, the federal government of Nigeria resulted into free food programme for elementary schools in the 36 States. This study aims at looking at the microbial loads of organisms present in the foods and its antibiotic sensitivity and how it can help the government in strategizing or re-strategizing their plans. A cross-sectional study was conducted in Ilorin metropolis within three local government areas (South, West, and East) with a total of 128 food samples. Microbiological assay were conducted on samples obtained from the elementary schools. The samples collected were cultured on three culture media (Blood agar, MacConkey agar and Saboroud Dextrose agar) and incubated at $37^{\circ} \mathrm{C}$. Characterization of isolates to specie level was done using their morphological appearance, colour of colonies, growth pattern, biochemical test and gram staining. Also antibiotic sensitivity test was also done using the disc diffusion method. Some of the organism isolated from food sampled were Staphylococcus epidermis, Proteus vulgaris, Streptococcus lactics, E. coli, and Candida albicans and they were resistant to Streptomycin, Cloxacilin, Ampicillin e.t.c and sensitive to Ofloxacin, Perfloxacin, Vancomycin e.t.c. Generally, this study reveals that out of the foods sold in the three local government, $30.7 \%$ of the foods are acceptable, $9.7 \%$ tolerable and $67.8 \%$ unacceptable according to the International Commission for Microbiological Specification for foods.
\end{abstract}

Keywords: Microbial Loads, Schools, Food Handlers

\section{Introduction}

Food is one of human's primary needs besides clothing and house. Food holds an important role in human's lives, thus a high level of food safety is needed to ensure that human is safe from diseases or dangers that come from foods Therefore, in the market food safety is a predictor for welleducated consumers to buy a certain food [1]. Though contamination could be from biological and chemical substances, most of foodborne diseases (90\%) are mainly caused by microbial contamination [2].

Food safety and quality is a public health concern especially the foods served to students and pupils at Schools by vendors.
Children in the African region usually experience five episodes of diarrhea per year and about 800,000 children die each year from diarrhea and dehydration. Diarrhea remains the third cause of death among children under age five, accounting for 10,000 deaths annually [3].

The global incidence of food borne disease is difficult to estimate, but it has been reported that in 2005 alone, 1.8 million people died from diarrheal diseases [4].

Therefore, within this study, we have decided to determine percentage occurrence of micro-organism present in the foods sold to pupils in some elementary schools in three Local Government Areas and their antibiotic sensitivity. Most schools in developed countries provide food for their pupils in school 
unlike Nigeria. Recently the present administration has passed the "provision of school foods to the pupils in government schools" into bill in which nine State practice it for now.

\section{Materials and Methods}

\subsection{Study Area}

The study area is the city of Ilorin which is the largest urban center in Kwara State. It is also the capital of Kwara State. Ilorin city occupies an area of $89 \mathrm{~km}^{2}$ and lies between the latitude $8^{\circ} 30^{\prime}$ North and longitude $4^{\circ} 35^{\prime}$ East. Ilorin metropolis is composed of three local government areas; Ilorin West, Ilorin East, and Ilorin South. The population of Ilorin is 777,667. According to the records of Kwara State Universal Basic Education (KWSUBEB), there are 346 registered private primary schools and 191 public primary schools in Ilorin.

\subsection{Sample Collection}

A total of 128 samples which comprises of white rice, jollof rice, wanke and white rice and spaghetti was collected. The food samples were bought into sterile plastic plate with covers, kept into a cold box containing ice packs and transported within an hour to Genesis laboratory at Ogbomosho Oyo State, for microbial analysis.

\subsection{Microbiological Analysis}

$10 \mathrm{ml}$ of distilled water was added to each food samples and mix to obtain homogenous mixture of samples. Sterile wire loop was used to pick a volume of $(0.1 \mathrm{ml})$ and transfer to the Medias in used (Macconkey Agar, Blood Agar and Saboroud Dextrose Agar). It was incubated for 24hrs and 4days and further microbial analysis carried out Colony count and Characterization.

\subsection{Characterization of Isolates}

Bacteria Isolate where identify to specie level using their morphological appearance color of colonies, growth pattern, biochemical test and their Gram Staining reactions, fungal isolated where viewed with $\mathrm{KOH}$ (potassium hydroxide) for microscopy.

\subsection{Antibiotics Sensitivity Test}

The Standardized disc diffusion method was used and the zone size interpreted in correlation to the one suggested by the National Committee for Clinical Laboratory Standards. The Following Antibiotics where used (Rapid Labs UK) Ofloxacin, Perfloxacin, Ciprofloxacin, Ceftriaxone, Cefuroxime, Ceftazidine, Levofloxacin, Streptomycin, Ampicillin, Augmentin, Cloxacillin, Erythromycin Gentamicin, Nitrofurantion, Tetracycline, and Vancomycin.

\subsection{Data Analysis Method}

Tables and charts will be used in analyzing the results such as bar chart and pie chart.

\section{Results}

\subsection{Sampled Foods}

The organism isolated from one hundred and twenty-eight food samples that were taken to the laboratory for analysis are as follow:

Table 1. Foods sampled and the organisms isolated in each food.

\begin{tabular}{lll}
\hline S/n & Food sampled & Microorganism isolated \\
\hline 1 & White rice & Staphylococcus epidermis, Proteus vulgaris, Bacillus cereus, Klebsiella pneumoniea, Staphylococcus aureus, \\
2 & Jollof rice & Streptococcus lactis, E. coli, Proteus mirabilis, Candida albicans \\
3 & Wanke & Proteus vulgaris, Bacillus cereus, Streptococcus spp., Staphylococcus epidermis, E. coli \\
4 & White rice and spaghetti & Klebsiella pneumoniea, Proteus mirabilis, Proteus vulgaris, Streptococcuslactis. \\
\hline
\end{tabular}

It has been revealed in Table 1 that the most common food sold in schools is rice but in different forms, such as white rice, jollof rice, wanke, rice and spaghetti and organisms isolated.

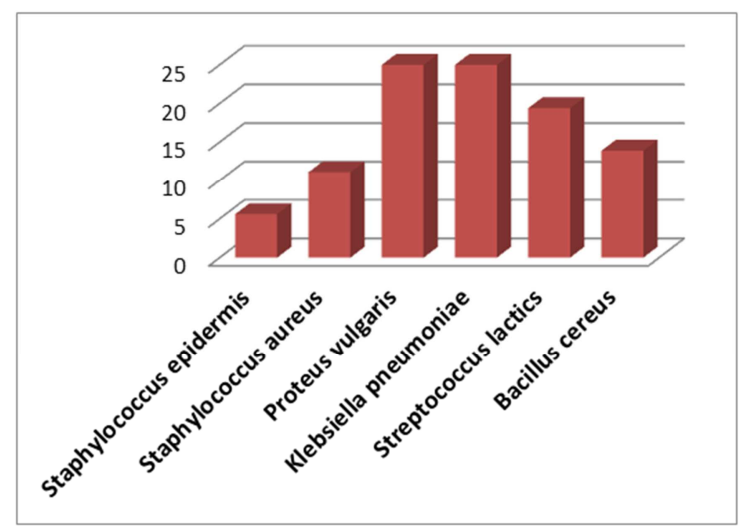

Figure 1. Percentage relative occurrence of each organism isolated from food sampled in Ilorin South such as Staphylococcus aureus, Proteus mirabilis, Klebsiella pneumonia e.t.c. 


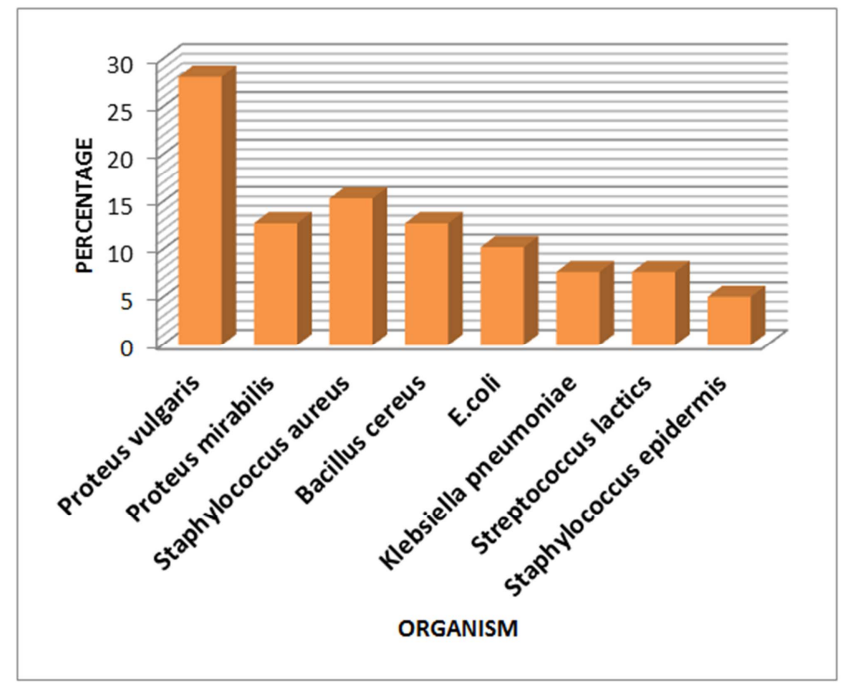

Figure 2. Percentage relative occurrence of each organism isolated from food sampled in Ilorin West such as Proteus vulgaris, proteus mirabilis, Bacillus cereus, Streptococcus spp e.t.c.

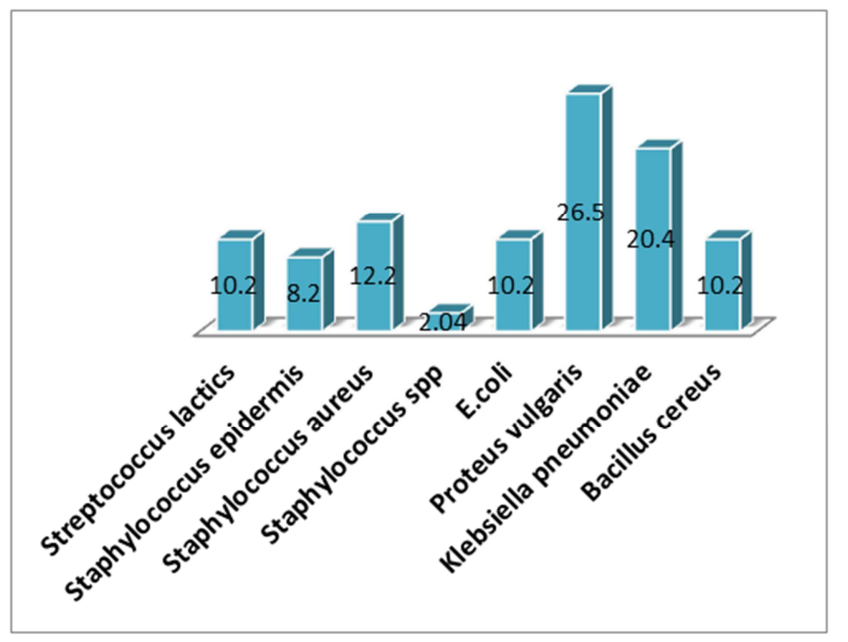

Figure 3. Percentage relative occurrence of each organism isolated from food sampled in Ilorin East such as Streptococcus lactic, Staphylococcus spp, E.coli, Bacillus cereus e.t.c.

\subsection{Antibiotic Sensitivity}

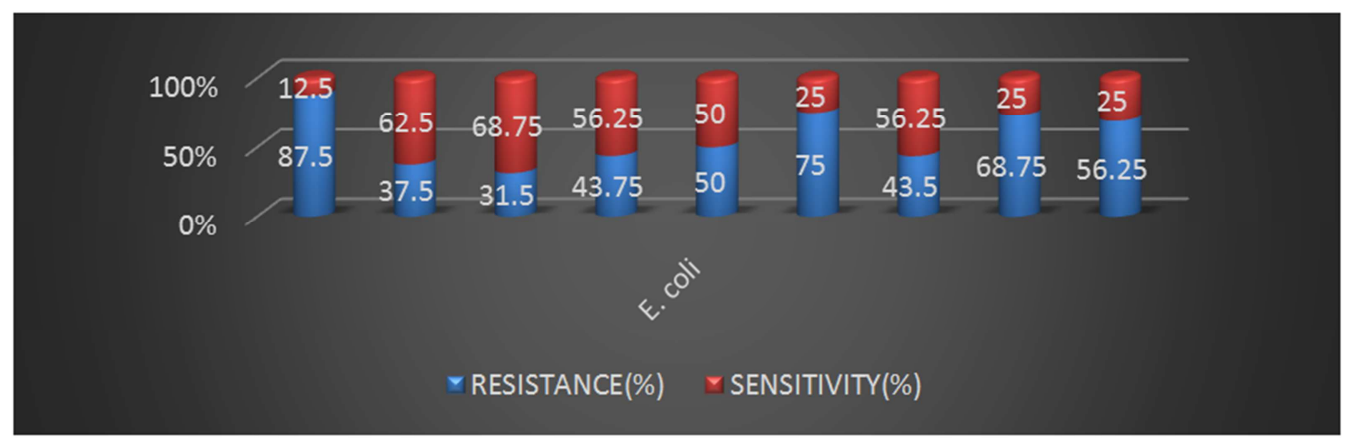

Figure 4. Percentage occurrence of sensitivity and resistance of microorganism isolated.

\section{Discussion}

In Ilorin South, the percentage occurrence of bacteria isolated are $5 \%$ of Staphylococcus epidermis which was resistant to 7 antibiotics $(43.75 \%)$ and sensitive to 9 antibiotics (56.25\%), 11\% of Staphylococcus aureus which was resistant to 6 antibiotics $(37.5 \%)$ and sensitive to 10 antibiotics $(62.5 \%), 25 \%$ of Proteus vulgaris was resistant to 9 antibiotics (56.25\%) and sensitive to 7 antibiotics (43.75\%), $25 \%$ of Klebsiella pneumonia which was resistant to 7 antibiotics (43.75\%) and sensitive to 9 antibiotics (56.25\%), $19.4 \%$ of Streptococcus lactis which was resistant to 5 antibiotics (31.25\%) and sensitive to 11 antibiotics $(68.75 \%)$, and $13.9 \%$ of Bacillus cereus which was resistant to 11 antibiotics (68.75\%) and sensitive to 5 antibiotics (31.25\%) while that of fungi is $100 \%$ of Candida albicans.

The percentage occurrence of bacteria isolated in Ilorin West, are $28.2 \%$ of Proteus vulgaris which was resistant to 9 antibiotics (56.25\%) and sensitive to 7 antibiotics (43.75\%), $12.8 \%$ of Proteus mirabilis which was resistant to 14 antibiotics $(87.5 \%)$ and sensitive to 2 antibiotics, (12.5\%),
$15.4 \%$ of Staphylococcus aureus which was resistant to 6 antibiotics (37.5\%) and sensitive to 10 antibiotics (62.5\%), $12.8 \%$ of Bacillus cereus was resistant to 11 antibiotics $(68.75 \%)$ and sensitive to 5 antibiotics $(31.25 \%), 10.3 \%$ of $\mathrm{E}$. coli which was resistant to 8 antibiotics (50\%) and sensitive to 8 antibiotics (50\%), 7.7\% of Klebsiella pneumonia which was resistant to 7 antibiotics $(43.75 \%)$ and sensitive to 9 antibiotics (56.25\%), 7.7\% Streptococcus lactic which was resistant to 5 antibiotics $(31.25 \%)$ and sensitive to 11 antibiotics $(68.75 \%)$ and $5.1 \%$ of Staphylococcus epidermis which was resistant to 7 antibiotics $(43.75 \%)$ and sensitive to 9 antibiotics $(56.25 \%$ )while in fungi isolates, no fungi was isolated.

While in Ilorin east, the percentage occurrence of bacteria isolated are $10.2 \%$ Streptococus lactic which was resistant to 5 antibiotics (31.25\%) and sensitive to 11 antibiotics (68.75\%), $8.2 \%$ Staphylococcus epidermis which was resistant to 7 antibiotics (43.75\%) and sensitive to 9 antibiotics (56.25\%), $12.2 \%$ of Staphylococcus aureus which was resistant to 6 antibiotics (37.5\%) and sensitive to 10 antibiotics (62.5\%), $2.04 \%$ of Staphylococcus spp which was resistant to 7 antibiotics (43.75\%) and sensitive to 9 antibiotics (56.25\%), 
$10.2 \%$ of E. coli which was resistant to 8 antibiotics (50\%) and sensitive to 8 antibiotics (50\%), 26.5\% of Proteus vulgaris which was resistant to 9 antibiotics $(56.25 \%)$ and sensitive to 7 antibiotics (43.75\%), 20.4\% of Klebsiella pneumonia which was resistant to 7 antibiotics $(43.75 \%)$ and sensitive to 9 antibiotics $(56.25 \%), 10.2 \%$ Bacillus cereus which was resistant to 11 antibiotics $(68.75 \%)$ and sensitive to 5 antibiotics (31.25\%)while that of fungi is $100 \%$ Candida albicans.

The occurrence of bacteria isolated in the three local government, was in conformance with a study done [5] and [6] and fungi isolated was not in line with the study.

[7] Also reported similar microorganisms from ready to eat rice sold in Benin City. The presence of indicator organisms, pathogens or high bacteria counts in food stuffs, food contact surfaces, equipment and utensils provides a direct and relevant measure of cleaning efficiency and hygiene [8]. Therefore, if large population of students is affected, this development will no doubt have negative impact on their performance in the school, due to absenteeism caused by food-borne illnesses. In some cases, it can lead to mortality, thereby creating psychological effects on the students, teachers and parents. This may also have ripple effects on the future of the state and the country in general.

Generally speaking, this study opined that all the foods provided to the students by the food vendors in all the schools are of unacceptable microbial loads because the International Commission for Microbiological Specification for Foods states that ready-to-eat foods with plate counts between $0-103$ is acceptable, between $104-\leq 105$ is tolerable and 106 and above is unacceptable.

\section{Conclusion}

Generally, it has been revealed that there is a significant difference in the microbial load content of foods sold in Basic Educational Service and it was observed that out of the foods sold in the three local governments, $30.7 \%$ of the foods are acceptable, $9.7 \%$ tolerable and $67.8 \%$ unacceptable.

The isolation of Bacillus cereus, Staphylococcus aureus, E. coli, Klebsiella spp, Proteus spp, Candida albicans, corroborate the findings [6] in which these organisms were isolated in ready- to- eat- foods. This is worrisome because of the public health impact of these microbes due to their pathogenic nature in suitable and susceptible host.

Contamination of foods could have resulted from inappropriate processing, incomplete heating, or secondary contamination via contact with contaminated equipment's and utensils. Although in this study, Salmonella and Shigella species were not isolated, nevertheless the presence of E. coli and other Enterobacteria is an indication of possible faecal contamination of food, water or food workers and poor hygienic processing practices [9], The presence of $S$. aureus is largely as a result of human contact and this suggests poor hygiene practices of the operators since this organism is a normal flora of the skin and nasal passage [10]. In addition, the presence of $S$. aureus, an enterotoxin producer which can cause serious gastroenteritis [11] and Ps. aeruginosa, an opportunistic pathogen, is known to cause food spoilage and can lead to economic loss [12] must be of outmost concern.

Thus Bacillus spp. Staphylococcus aureus commonly from man, thus there higher percentage prevalence is therefore not out of order [13].

It is mandatory that foods must be free from contaminations as much as possible. The presence of E. coli, $S$. aureus and $B$. cereus demonstrates a potential health risk as these organisms are pathogenic and have been implicated in food borne diseases [14]. Food borne illness can be prevented by good hygiene practices such as the use of Good Manufacturing Practices (GMP) and Hazard Analysis Critical Control Point (HACCP) application in the chain of food production, chain of custody and processing. To curb this Education of the food handlers/food vendors on food safety practices and a close and stringent supervision of ready-to-eat foods sold in the schools should be carried out by relevant authorities to prevent food borne illness.

\section{References}

[1] Centre for Disease Control on National outbreak reporting system 2009-2016.

[2] Purtiantini. 2010. The relationship between knowledge and attitude for choosing school kids foods at SDIT

Muhammadiyah Al Kautsar Gumpang Kartasura. Journal of Health Science [e-journal] http://etd. eprints.ums.ac.id/9535/2/J310080049.pdf (accessed 17 June 2016)

[3] State fact sheets. (2015). Retrieved from $\mathrm{http}: / / w w w . e r s . u s d a . g o v /$ data-products/state-factsheets $/$ statedata.asp $x$ StateFIPS $=05 \&$ StateName $=$ Arkansas

[4] World Health Organization, (2015). Food borne diseases; a focus for health education. World Health Day.

[5] Oranusi, U. S., and Braide, W. (2013). A study of microbial safety of ready-to-eat foods vended on highways: OnitshaOwerri, south east Nigeria. International Research Journal of Microbiology 3(2): 066-071.

[6] Legesse G., Zenabu Hs., Zelalem A., Reta T., and Bidir Z., Antimicrobial Resistance and Infection Control 2015 BMC part of spring nature.

[7] Wogul, A. B. (2011). Epidemiology of food poisoning outbreaks in Singapore, 2001- 2005. Epidemiological News Bulletin, Journal of Microbiology, 31: 68-72.

[8] Yan Z., Luqi Z., Yating Z., Pingang He, Qingjiang Wang (2018). Simultaneous detection of three foodborne pathogenic bacteria in food samples by microchip capillary electrophoresis in combinationwith polymerase chain reaction Journal of Chromatography A Volume 1555, 22 June 2018, Pages 100-105.

[9] Tambekar, D. H., Shirsat, S. D., Suradkar, S. B., Rajankar, P. N., and Banginwar, Y. S. (2007). Prevention of transmission of infectious disease: Studies on hand hygiene in health-care among students. Continental Journal of Biomedical Sciences, 1: 6-10. 
[10] Mekonnen H, Habtamu T, Kelali A, Shewit K (2012). Food safety knowledge and practices of abattoir and butchery shops and the microbial profile of meat in Mekelle City, Ethiopia. Asian Pac. J. Trop. Biomed. 12:952-957.

[11] Taye M, Berhanu T, Berhanu Y, Tamiru F, Terefe D (2013). Study on carcass contaminating E. coli in apparently healthy slaughtered cattle in Haramaya University slaughter house with special emphasis on E. coli O157:H7, Ethiopia. J. Vet. Sci. Technol. 4:132.

[12] Liao, C. H., 20015. Food Spoilage Microorganisms. In: Pseudomonas and Related Genera, Blackburn, C. W. (Ed.). Woodhead Publishing Ltd., Cambridge, UK.
[13] Elbagory AM, Hammad AM, Alzahra SMA. Prevalence of Coliforms, antibiotic resistant Coliforms and E. coliserotypes in raw milk and some varieties of raw milk cheese in Egypt. Nutr Food Technol. 2016;2(1) https://doi.org/10.16966/24706086.114

[14] 17th Asia-Pacific International Symposium on Microscale Separation and Analysis (APCE 2017), 10-13 November 2017, Shanghai, China. 